\title{
Dietary Glycaemic Index and Type 2 Diabetes Mellitus: Potential Modulation of Gut Microbiota
}

\author{
Hanusha Durganaudu, Thubasni Kunasegaran, Amutha Ramadas* \\ Jeffrey Cheah School of Medicine \& Health Sciences, Monash University Malaysia, Jalan Lagoon Selatan, 47500 Bandar \\ Sunway, Selangor Darul Ehsan, Malaysia
}

\begin{abstract}
Diet therapy is often the first-line approach in prevention and management of type 2 diabetes mellitus (T2DM). Adoption of low glycaemic index (GI) diet one of the recent dietary strategies to modulate glycaemic response in individuals with T2DM. Generally, diet has strong influence on the gut microbiota, which recently have been found to be associated with insulin resistance and the inflammatory response in diabetes. The possible modulation of the gut microbiota with dietary intervention is a topic of emerging interest, with limited evidence among T2DM population. In this review, we have narrated the available evidence and discussed the current knowledge about diet manipulation associated with dietary GI in order to shape the gut microbiota. As a conclusion, we have pointed out several key research directions that may have helpful impact on diet interventions with modulation of gut microbiota on the pathogenesis and therapeutic implications in T2DM.
\end{abstract}

Keywords: microbiome; type 2 diabetes mellitus; carbohydrate-glycaemic index; nutrition

Received: $10^{\text {th }}$ April 2020

Accepted: $12^{\text {th }}$ May 2020

Published Online: $19^{\text {th }}$ May 2020
*Correspondence: Amutha Ramadas, Jeffrey Cheah School of Medicine \& Health Sciences, Monash University Malaysia 47500, Bandar Sunway, Selangor Darul Ehsan, Malaysia; amutha.ramadas@monash.edu

Citation: Durganaudu H, Kunasegaran T and Ramadas A. Dietary Glycaemic Index and Type 2 Diabetes Mellitus: Potential Modulation of Gut Microbiota. Prog Microbes Mol Biol 2020; 3(1): a0000082. https://doi.org/10.3687/pmmb.a0000082

\section{INTRODUCTION}

Diabetes mellitus is defined as a "metabolic disorder of multiple aetiology characterised by chronic hyperglycaemia with disturbances of carbohydrate, fat and protein metabolism resulting from defects in insulin secretion, insulin action, or both"[1]. Type 2 diabetes mellitus (T2DM) is the most common form of diabetes, accounting for about $80 \%$ of all diabetes cases mostly after the age of $30^{[2]}$. T2DM is characterised by three basic abnormalities - insulin resistance, impaired insulin secretion and increased hepatic glucose production, either of which may be the predominant feature ${ }^{[3,4]}$. This results in a disorder of carbohydrate metabolism, and fat, protein and mineral metabolism also can be affected.

The World Health Organization has defined the diagnostic criteria for diabetes through a single raised glucose reading with symptoms, otherwise raised values on two occasions, of either fasting plasma glucose $\geq 7.0 \mathrm{mmol} / 1(126 \mathrm{mg} / \mathrm{dl})$ or with a glucose tolerance test, two hours after the oral dose a plasma glucose $\geq 11.1 \mathrm{mmol} / \mathrm{l}(200 \mathrm{mg} / \mathrm{dl})^{[1]}$. In diabetes management, nearly all T2DM related research outcomes are looking at reduction in glycosylated haemoglobin (HbA1c) and report from the UK Prospective Diabetes Study (UKPDS) has recommended $\mathrm{HbAlc}$ to be maintained below $7.1 \%$ to minimise T2DM-related complications $^{[5]}$.
It has been shown that individuals from primitive societies, where the incidence of diabetes is low who then move to societies where food is too readily available, often progress to develop diabetes ${ }^{[6]}$. Although many people with T2DM could be managed by dietary modification alone, eventually they may require insulin therapy due to its progressive nature.

Although T2DM could be inherited, modifiable factors such as body composition and nutrition play important roles in the etiology of T2 $\mathrm{DM}^{[7]}$. The goals of Medical Nutrition Therapy (MNT) in people with diabetes are to achieve and maintain normal blood glucose, lipid and lipoprotein and blood pressure levels by addressing the nutritional needs and maintaining the pleasure of eating which eventually, prevent or slow the development of complication $^{[8,9] \text {. }}$

One of such dietary modifications that many MNTs recommend will be adoption of low glycaemic index (GI) diet. GI ranks carbohydrate-containing foods according to their effect on postprandial glycaemia ${ }^{[10,11]}$. GI is a measure of the increase in blood glucose two hours after consumption of the food of interest, with reference to glucose or white bread. Glycaemic load (GL), a product of GI and amount of carbohydrate in the food, is another measure of glycaemic response to carbohydrate-containing foods and could improve the 
evaluation of glycaemic response to a diet ${ }^{[12]}$. Foods with low GI such as parboiled rice, barley, oats and legumes lower the postprandial hyperglycaemia, while high GI foods such as white bread, potatoes, white rice and commercial breakfast cereals will show the opposite effect $^{[8,13]}$.

Additionally, increasing evidence has highlighted that the role of gut microbiota in a multitude of human illnesses, both inside and outside of gut, including irritable bowel syndrome ${ }^{[14]}$, colorectal cancer ${ }^{[15,16]}$, skin diseases ${ }^{[17,18]}$, neurological-related diseases ${ }^{[19,20]}$. Similarly, there are also emerging findings on the strong association between development of diabetes and gut microbiota $^{[21,22]}$. Gut microbiota is a dynamic identity that can result in various pathophysiological changes in human's internal environment, though the composition changes gradually over the lifetime ${ }^{[23,24]}$. Gut microbiota plays a critical role in several metabolic functions namely amino-acid synthesis, absorption of dietary fats and fatsoluble vitamins, production of short-chain fatty acids (SCFAs), activation of glucose homeostasis, lipid energy metabolism, calorie removal and regulating bile acid transformation among others ${ }^{[25]}$. This may predispose or protect an individual against diabetes.

As gut microbiota can be easily influenced by environmental factors especially one's dietary intake, there is a potential for gut microbiota to act as a modulator of dietary GI and T2DM relationship. In this review we will be summarizing the evidence associating dietary GI and T2DM, and subsequently discuss the potential role of gut microbiota in modulating this relationship.

\section{DIETARY GLYCAEMIC INDEX AND DIABETES}

A cross-sectional study in South Italy found a dosedependent relationship between dietary GI and GL with $\mathrm{HbA1c}$, where patients with diabetes with highest GI and GL had the highest HbA1c levels ${ }^{[26]}$. GI-based dietary education has been suggested to be a useful tool in diabetes management ${ }^{[27,28]}$. Miller and Gutschall[ ${ }^{[29]}$ reported a nine-week nutrition education regarding GI and GL which improved dietary intake, knowledge, outcome and efficacy expectations and empowerment for diabetes management. Although GI could lead to a better dietary intake in people with diabetes, only few organisations recommended the use of low GI diets ${ }^{[30-32]}$.

Retrospective study by Burani et al. ${ }^{[26]}$ found a reduction in $\mathrm{HbA} 1 \mathrm{c}$ of $19 \%$ and body mass index (BMI) of $8 \%$ following inclusion of low GI diet in lifestyle intervention, which was well accepted by the participants. Several trials have supported the role of GI in glycaemic control in patients with $\mathrm{T}_{2} \mathrm{DM}^{[34]}$. Significant improvements have been seen in $\mathrm{HbA1c}$ and/or $\mathrm{FPG}^{[34-37]}$, insulin sensitivity ${ }^{[34]}$ and serum fructosamine ${ }^{[36]}$ with low GI diets. The role of GI diets in glycaemic control has been confirmed by several meta-analyses and reviews ${ }^{[13,38,39]}$. However, there is a lack of studies on the technology assisted low GI interventions, and only one feasibility nutritionist-delivered, PDA-assisted low-GI dietary intervention by Ma et al. ${ }^{[40]}$ has been discovered.
Low GI diets also have been shown to have favourable impact on lipid profile and reduction in cardiovascular risk of patients with $\mathrm{T}_{2} \mathrm{DM}^{[35,41-44]}$. Studies have reported significant decrease in total cholesterol and LDL-C with prescribed low GI diets ${ }^{[34]}$. Longer educational programmes to improve diet quality by emphasizing low GI diets also have shown to lower the LDL-C ${ }^{[45,46]}$ and increase the HDL-C ${ }^{[37]}$. Besides the cholesterol levels, a low GI or GL diet may be preferred for the dietary management of T2DM because of sustained reductions in postprandial glucose and c-reactive protein ${ }^{[47]}$ and the increase in the plasma adiponection concentrations ${ }^{[48]}$.

There is also evidence showing the positive impact of low GI diet on other health outcomes in people with diabetes. Low GI diet accompanied with exercise programme was found to improve cardiovascular health ${ }^{[49]}$ and protect against-induced hypoglycaemia in T2DM patients ${ }^{[50]}$. Low GI diet could assist with the weight management programme in patients with diabetes ${ }^{[43,46]}$. Yusoff and colleagues $^{[36]}$ reported a significant reduction in waist circumference in Asian patients after 4 months of following a low GI diet. Low GI diet has also generally results in better cognitive performance in the postprandial period in adults with T2DM and reduce their dependency on diabetes medication ${ }^{[45,51]}$.

Interestingly, there were studies which did not support the role of GI in diabetes management ${ }^{[52]}$. In one of such studies, low GI diet with calorie restriction in overweight patients with T2DM did not find any significant reduction in $\mathrm{HbA} 1 \mathrm{c}^{[46]}$. Data derived from the Atherosclerosis Risk in Communities study suggest high GL intake to be a CHD risk factor only among Whites without diabetes and not in individuals with diabetes ${ }^{[53]}$. Cheong et al. ${ }^{[54]}$ concluded addition of a low-GI component to a walking did not improve anthropometric or metabolic outcomes in diabetic patients. A review by Barojek and Morello ${ }^{[55]}$ has identified short coming in terms of power of the study and confounders, which could have affected otherwise positive findings in these studies.

As low GI foods are generally rich in fiber and other nutrients, the consumption of this diet has been shown beneficial to diabetic patients ${ }^{[56]}$. Incorporation of such foods in every day diet may be an effective approach for weight management, glycaemic control and favourable lipid profile. However, the concept of GI should not be used in isolation, but to be used as an adjunct treatment to existing lifestyle management of T2DM in fine-tuning the glycaemic control ${ }^{[45,57]}$.

\section{DIETARY GLYCAEMIC INDEX AND GUT MICROBIOTA}

Exploration of a potential association between dietary GI and changes in the proportion of certain gut microbiota is relatively a newer research area, with limited evidence among T2DM population.

An experimental study among individuals at risk of metabolic syndrome showed largest increase in the Bifidobacterium spp. (an established gut health biomarker) level in high carbohydrate/high glycaemic index $(\mathrm{HC} /$ HGI) group compared to control group ${ }^{[58]}$. This change was 
associated with reduced fasting glucose, fasted insulin and cholesterol levels compared to baseline. Furthermore, HC/HGI group was also associated with increased Bacteroides numbers as well as reduction in body weight, BMI and waist circumference. Both Bacteroides and Bifidobacterium spp. have been independently associated with reduction in risk factors for metabolic syndrome and improved body energy regulation in the past ${ }^{[59]}$. Interestingly, the study found an increased abundance of Faecalibacterium prausnitzii with both high saturated fat (HS) diet and high carbohydrate/low glycaemic index (HC/LGI) diets. HS group also experienced an increase in faecal SCFA concentration. However, faecal acetate percentage may be inversely correlated with absorbed acetate percentage (after rectal infusion), as demonstrated by Vogt and Wolever ${ }^{[60]}$. In that case, higher SCFA noted in the HS group may be due to decreased absorption, instead of higher colonic fermentation.

A study exploring specific foods with lower GI responses in vitro, found minimally processed wholegrain cereals such as wholegrain oats and granola resulted in significant growth in the friendly bacteria namely Bifidobacterium genus and Lactobacillus-Enterococcus group $^{[61]}$. Wholegrains with minimal processing also resulted in increase in Atopobium cluster and BacteroidesPrevotella group after 10 hours. Increase in Clostridium histolyticum after instant porridge fermentation (highly processed wholegrains) is also noted to be significantly higher compared to the decrease observed in minimally processed wholegrains.

Another low GI grains that were investigated in the past were barley grains. An in vivo study found barley intake lead to increased abundance of Prevotella and Lactobacillus, as well as Candidatus homeothermaceae in obese and lean mice ${ }^{[62]}$. Barley intake was also linked with lower levels of plasma insulin and resistin, a cysteine-rich peptide secreted by adipocytes, immune cells, and epithelial cells which are found in higher levels in metabolic syndrome cases ${ }^{[63]}$.

Another study utilised models such as static in vitro digestion and dynamic gastric model to simulate the normal digestion process in investigating relationship between different types of barley grains and microbiota ${ }^{[64]}$. During early stage of digestion in the study, it noted that the digesta of wild-type barley Hordeum vulgare cv Golden Promise (Hv) and Amylose-only (AO) breads portrayed higher abundancy of Firmicutes, whereas wheat and wild barley Hordeum vulgare subsp. Spontaneum (Hs) grain bread digesta contained lower levels of it. Hs grain bread digesta demonstrated increased abundance of actinobacteria (30-fold higher than control) to the detriment of Bacteroidetes and Firmicutes. However, samples representing later stages of digestion behaved differently as Bacteroidetes and Actinobacteria were found to be abundant in all digesta. Specifically, increased Actinobacteria was noted in AO fermentation (compared to early digestion stage samples) while Bacteroidetes was more abundant in fermentation of Hs grain digesta. Aside from that, Proteobacteria levels in wheat bread digesta fluctuated from early digestion to late digestion stage. Therefore, these results portray the potential of low GI barley-based bread in regulating gut microbiota.

\section{ROLE OF GUT MICROBIOTA IN TYPE 2 DIABETES}

Several studies have investigated and showed some forms of association between gut microbiota and development of T2DM. The role of gut microbiota may be evaluated in several aspects.

Insulin resistance in T2DM patients may have resulted from increased production of hepatic triglyceride facilitated by Firmicutes and Bacteroidetes, as they enhance the monosaccharide uptake from the host gut ${ }^{[65]}$. Aside from the impact on carbohydrate metabolism, high ratios of Firmicutes to Bacteroidetes is also found to alter the production of SCFAs with an increase in acetate production and decrease in butyrate production ${ }^{[66]}$. The increased levels of acetate in the blood is found to result in insulin resistance and heightened production of ghrelin (an-appetite stimulating hormone) in the stomach, as illustrated by a recent study among individuals with metabolic syndrome ${ }^{[67]}$. On the other hand, decrease in butyrate levels also encourage insulin resistance through promotion of low-level inflammation ${ }^{[68]}$.

Aside from the diversity of gut microbiota, another aspect that could be looked into is its role in facilitating immune response which lead towards development of T2DM. Abundance of Prevotella bacterial species was found particularly in obese T2DM individuals, and known to increase the levels of pro-inflammatory cytokines, besides encouraging low-grade inflammation and insulin resistance ${ }^{[69]}$. Verrucomicrobia, on the other hand, which are known to contribute towards the maintenance of antiinflammatory state of gut and improve insulin sensitivity were found less in T2DM individuals in a study conducted in Pakistan ${ }^{[70]}$. The same study also found an increase in the levels of gram-negative bacteria such as Dialister and Allisonella, which may have contributed to the rise in levels of lipopolysaccharide (LPS), which eventually binds with CD14 and mediates inflammatory response. Another class of bacteria which is found to be abundant among people with diabetes is Fusobacteria, which plays critical role in inflammatory responses, mounting adhesiveness to host epithelial cells and energy generation ${ }^{[71,72]}$.

Furthermore, the link between gut microbiota and T2DM can also be explored by looking into their association with bile acids. It is well-known that one of the important symbiotic roles played by gut microbiota is in terms of bile acid transformation, as their composition has been found to affect the concentration and composition of circulating bile acids ${ }^{[73]}$. This in turn affects the individual's risk in developing obesity and related disorders. Most of the bile acid biotransformation occurs in the large intestine which is extremely rich in microbiota, through a complex process ${ }^{[74]}$. This is one process in which the role of gut bacteria can be directly observed, as it is catalysed by bile salt hydrolase (BSH), an enzyme which is present in several gut bacteria such as Clostridium, Bacteroides, Lactobacillus, Bifidobacterium and Enterococcus ${ }^{[75]}$. Bile acids in the blood circulation play a role in homeostasis of carbohydrates and lipids, further reinforcing their importance as regulatory molecules in that aspect ${ }^{[73]}$. Bile acids also pose some direct 
antimicrobial action which subsequently impacts the survival and colonisation of certain gut microbiota ${ }^{[76]}$.

As an individual's food consumptions affect both gut microbiota and development of diabetes, further exploration can be made to investigate the changes in gut microbiota according to the type of prescribed dietary pattern, and its impact on diabetes development and/or severity.

\section{CONCLUSION}

The search for the optimal nutritional strategy in T2DM patients remains an unresolved issue. It is important that the potential benefits of suitable GI diet and the T2DM patient's microbiota, which in turn will impact on the progression of disease complication, are taken into consideration. In this review, we have provided evidence that different dietary patterns have different impacts on gut microbial composition. These findings suggest that the gut microbiota contribute to the pathophysiological regulation of glucose tolerance, insulin secretion and in inflammation.

Hence, future studies should define the features of the gut microbiome in diet consumption that contribute to the T2DM in defined populations. Furthermore, with the emerging advances in technology, the relationship between important biomarkers to changes in gut microbiota and microbiota metabolites modulated by recommended diets are worth investigating in future studies to aid prevent or treat diabetes-related disorders in a strategic manner. In addition, it is also important to focus on bile stress and its effects on the gut microbiota in future direction in finding therapeutic strategies to reduce the aggregate metabolic burden in human populations. Some bacteria can use bile as their host to regulate virulence determinants and produce secondary bile acids that regulate the normal homeostasis of tissues in human body which leads to unwanted complications. Thus, further studies should investigate how bacteria sense bile and regulate it response it induces and reveal the effect of different bile acid profiles in host tissues by virulence factor production. Lastly, approaches to modulate the microbiome-bile acid formation through diet may likely reduce the risk and/or treat metabolic diseases and this need further investigation.

\section{Author Contributions}

The review and manuscript writing were performed by $\mathrm{HD}, \mathrm{TK}$ and AR.

\section{Conflict of Interest}

The authors declare that there is no conflict of interest in this work.

\section{References}

1. World Health Organisation. Definition and diagnosis of diabetes mellitus and intermediate hyperglycemia: report of a WHO/IDF consultation 2006

2. McDowell JR, Matthews DM and Brown FJ. What is diabetes? A handbook for the Primary Healthcare Team: Second Edition. Elsevier; Churchill Livingstone UK 2007.

3. Kahn BB. Type 2 diabetes: When insulin secretion fails to compensate for insulin resistance. Cell 1998; 92(5): 593-596.

4. Taylor R. Causation of type 2 diabetes - the gordian knot unravels. N Engl J Med 2004; 350(7): 639-641.

5. United Kingdom Prospective Diabetes Study (UKPDS) Group. Intensive blood-glucose control with sulphonylureas or insulin compared with conventional treatment and risk of complications in patients with type 2 diabetes (UKPDS 33). Lancet 1998; 352(9131): 837-853.

6. Diamond J. The double puzzle of diabetes. Nature 2003; 423(6940): 599-602.

7. World Health Organisation. Diabetes mellitus: Report of a WHO Expert Committee. Geneva 1965.

8. American Diabetes Association. Nutrition recommendations and interventions for diabetes: A position statement of the American Diabetes Association. Diabetes Care 2008; 31(1): S61-78.

9. Pegklidou KI, Nicolaou and Demopoulos VJ. Nutritional overview on the management of type 2 diabetes and the prevention of its complications. Curr Diabetes Rev 2010; 6(6): 400-409.

10. Jenkins DJ, Wolever TM, Taylor RH, et al. Glycemic index of foods: a physiological basis for carbohydrate exchange. Am J Clin Nutr 1981; 34(3): 362-366.

11. Jenkins DJ, Kendall CWC, Augustin LSA, et al. Glycemic index: overview of implications in health and disease. Am J Clin Nutr 2002; 76(1): 266S-273S

12. Sheard NF, Clark NC, Brand-Miller JC, et al. Dietary carbohydrate (amount and type) in the prevention and management of diabetes: A statement by the American Diabetes Association. Diabetes Care 2004; 27(9): 2266-2272.

13. Brand-Miller J, Hayne S, Petocz P, et al. Low-glycemic index diets in the management of diabetes. Diabetes Care 2003; 26(8): 2261-2267.

14. Selvaraj SM, Wong SH, Ser H-L, et al. Role of low FODMAP diet and probiotics on gut microbiota in irritable bowel disease (IBS). Prog Microbes Mol Biol 2020; 3(1): a0000069.

15. Lee LH, Ser HL, Ab Mutalib NS, et al. Winning the war against colon cancer chemo-preventive potential of novel streptomyces species derived from mangrove forst in Malaysia. Gut 2018; 67: A13-A14.

16. Lee LH, Law JWF, Khan TM, et al. Unveiling the anti-colon cancer potential of Sarawak mangrove-derived novel streptomycetes. Gut 2019; 68: A42-A43.

17. Lee LH, Letchumanan V, Khan TM, et al. Dissecting the gut and skin: budding association between gut microbiome in the development to psoriasis?. Gut 2019; 68: A41-A41.

18. Lee LH, Letchumanan, Khan TM, et al. Role of human microbiota in skin dermatitis and eczema: A systematic review. 2018; 67: A19-A19. Lee LH, Ser HL, Khan TM, et al. Relationship between autism and gut microbiome: current status and update. Gut 2019; 68: A40-A41.

20. Lee LH, Ser HL, Khan TM, et al. Dissecting the gut and brain: potential links between gut microbiota in development of alzheimer's disease? Gut 2018; 67: A18-A18

21. Gurung M, Li Z, You H, et al. Role of gut microbiota in type 2 diabetes pathophysiology. EBioMedicine 2020; 51: p. 102590

22. Dedrick S, Sundaresh B, Huang Q, et al. The role of gut microbiota and environmental factors in type 1 diabetes pathogenesis. Front Endocrinol 2020; 11(78)

23. O'Toole PW and Jeffery IB. Gut microbiota and aging. Sci 2015; 350(6265): 1214-1215.

24. Donaldson G.P, Lee SM and Mazmanian SK. Gut biogeography of the bacterial microbiota. Nat Rev Microbiol 2016; 14(1): 20-32.

25. Blandino G, Inturri R, Lazzara F, et al. Impact of gut microbiota on diabetes mellitus. Diabetes Metab 2016; 42(5): 303-315.

26. Esposito K, Maiorino MI, Palo CD, et al. Dietary glycemic index and glycemic load are associated with metabolic control in type 2 diabetes: The CAPRI experience. Metab Syndr Relat Disord 2010; 8(3): 255261.

27. Amano Y, Sugiyama M, Lee JS, et al. Glycemic index-based nutritional education improves blood glucose control in Japanese adults: A randomized controlled trial. Diabetes Care 2007; 30(7):1874-1876.

28. Gutschall M, Miller CK, Mitchell DC, et al. A randomized behavioural trial targeting glycaemic index improves dietary, weight and metabolic outcomes in patients with type 2 diabetes. Public Health Nutr 2009; 12(10): 1846-1854.

29. Miller CK and Gutschall M. A randomized trial about glycemic index and glycemic load improves outcomes among adults with type 2 diabetes. Health Educ Behav 2009; 36(3): 615-626.

30. Canadian Diabetes Association. Clinical practice guidelines for the prevention and management of diabetes in Canada. Can J Diabetes 2008; 32(1): S1-S201.

31. Nutrition Subcommittee of the Diabetes Care Advisory Committee of Diabetes. The implementation of nutritional advice for people with diabetes. UK. Diabet Med 2003; 20(10): 786-807.

32. Ministry of Health and Academy of Medicine of Malaysia. Malaysian clinical practice guidelines: management of type 2 diabetes mellitus (4th Edition). 2009. 
33. Burani $\mathrm{J}$ and Longo PJ. Low-glycemic index carbohydrates: an effective behavioral change for glycemic control and weight management in patients with type 1 and 2 diabetes. Diabetes Educ 2006; 32(1): p. 78(11)

34. Rizkalla SW, Taghrid L, Laromiguiere M, et al. Improved plasma glucose control, whole-body glucose utilization, and lipid profile on a low-glycemic index diet in type 2 diabetic men. Diabetes Care 2004; 27(8): 1866-1872

35. Kabir M, Jean-Micheal O, Vidal H, et al. Four-week low-glycemic index breakfast with a modest amount of soluble fibers in type 2 diabetic men. Metab 2002; 51(7): 819-826.

36. Yusof BNM, Talib RA, Kamaruddin NA et al A low-GI diet is associated with a short-term improvement of glycaemic control in Asian patients with type 2 diabetes. Diabetes Obes Metab 2009; 11(4): 387-396.

37. Jenkins DJA, Kendal CWC, McKeown-Eyssesn G, et al. Effect of a low-glycemic index or a high-cereal fiber diet on type 2 diabetes. JAMA 2008; 300(23): 2742-2753.

38. Thomas DE and Elliott EJ. The use of low-glycaemic index diets in diabetes control. Br J Nutr 2010; 104: 797-802.

39. Psaltopoulou T, Ilias I and Alevizaki M. The role of diet and lifestyle in primary, secondary, and tertiary diabetes prevention: A review of meta-analyses. Rev Diabet Stud 2010; 7(1): p. 26-35.

40. Ma Y, Olendzki BC, Rosal M, et al. PDA-assisted low glycemic index dietary intervention for type II diabetes: A pilot study. Eur J Clin Nutr 2006; 60(10): p. 1235.

41. Jimenez-Cruz A, Bacardi-Gascon M, Turnbull WH, et al. A flexible, low-glycemic index Mexican-style diet in overweight and obese subjects with type 2 diabetes improves metabolic parameters during a 6-week treatment period. Diabetes Care 2003; 26(7): 1967-1970.

42. Jiménez-Cruz A, Bacardi-Gascon M, Turnbull WH, et al. A high-fiber, moderate-glycemic-index, Mexican style diet improves dyslipidemia in individuals with type 2 diabetes. Nutr Res 2004; 24(1): 19-27.

43. Thomas DE, Elliott EJ and Baur L. Low glycaemic index or low glycaemic load diets for overweight and obesity. Cochrane Database Syst Rev 2009; 1 .

44. Opperman M, Venter CS and Oosthuizen W. Some health benefits of low glycaemic index diets-A systematic review. South African J Clin Nutr 2005; 18(3): 214-221.

45. Ma Y, Olendzki BC, Meriam PA, et al A randomized clinical trial comparing low-glycemic index versus ADA dietary education among individuals with type 2 diabetes. Nutrition 2008; 24(1): 45-56.

46. Heilbronn LK, Noakes M and Clifton PM. The effect of high- and low-glycemic index energy restricted diets on plasma lipid and glucose profiles in type 2 diabetic subjects with varying gycemic control. J Am Coll Nutr 2002; 21(2): 120-127.

47. Wolever T, Mehling C, Chiasson JL, et al. Low glycaemic index diet and disposition index in type 2 diabetes (the Canadian trial of carbohydrates in diabetes): A randomised controlled trial. Diabetologia 2008; 51(9): 1607-1615.

48. Qi L, Rimm E, Liu S, et al. Dietary glycemic index, glycemic load, cereal fiber, and plasma adiponectin concentration in diabetic men. Diabetes Care 2005; 28(5): 1022-1028.

49. Johnson ST, Bell GJ, McCarger LJ, et al. Improved cardiovascular health following a progressive walking and dietary intervention for type 2 diabetes. Diabetes Obes Metab 2009; 11(9): 836-843.

50. Ferland A, Brassard P, Lemieux S, et al. Impact of high-fat/lowcarbohydrate, high-, low-glycaemic index or low-caloric meals on glucose regulation during aerobic exercise in type 2 diabetes. Diabet Med 2009; 26(6): 589-595.

51. Papanikolaou Y, Palmer H, Binns MA, et al. Better cognitive performance following a low-glycaemic-index compared with a highglycaemic-index carbohydrate meal in adults with type 2 diabetes. Diabetologia 2006; 49(5): 855-862

52. Franz MJ. The glycemic index. Diabetes Care 2003; 26(8): 2466 2468.

53. Hardy DS, Hoelscher DM, Aragaki C, et al. Association of glycemic index and glycemic load with risk of incident coronary heart disease among Whites and African Americans with and without type 2 diabetes: the atherosclerosis risk in communities study. Ann Epidemiol 2010; 20(8): 610-616.

54. Cheong SH, McCarger LJ, Paty BW, et al. The first step first bite program: guidance to increase physical activity and daily intake of lowglycemic index foods. J Am Diet Assoc 2009; 109(8): 1411-1416.

55. Bajorek SA and Morello CM. Effects of dietary fiber and low glycemic index diet on glucose control in subjects with type 2 diabetes mellitus. Ann Pharmacother 2010; 44(11): 1786-1792.

56. Davis N, Forbes B and Wylie-Rosett J. Nutritional strategies in type 2 diabetes mellitus. Mt Sinai J Med 2009; 76(3): 257-268.

57. Riccardi G, Rivellese AA and Giacco R. Role of glycemic index and glycemic load in the healthy state, in prediabetes, and in diabetes. Am J Clin Nutr 2008; 87(1): 269S-274S.

58. Fava F, Gitau F, Griffin BA, et al. The type and quantity of dietary fat and carbohydrate alter faecal microbiome and short-chain fatty acid excretion in a metabolic syndrome 'at-risk' population. Int J Obes 2013; 37(2): 216-223.

59. Cani PD, Neyrinck AM, Fava F, et al. Selective increases of bifidobacteria in gut microflora improve high-fat-diet-induced diabetes in mice through a mechanism associated with endotoxaemia. Diabetologia 2007; 50(11): 2374-2383.

60. Vogt JA and Wolever TMS. Fecal acetate is inversely related to acetate absorption from the human rectum and distal colon. J Nutr 2003; 133(10): 3145-3148.

61. Connolly ML, Tuohy KM and Lovegrove JA. Wholegrain oat-based cereals have prebiotic potential and low glycaemic index. Br J Nutr 2012; 108(12): 2198-206.

62. Garcia-Mazcorro JF, Mills DA, Murphy K, et al. Effect of barley supplementation on the fecal microbiota, caecal biochemistry, and key biomarkers of obesity and inflammation in obese $\mathrm{db} / \mathrm{db}$ mice. Eur J Nut 2018; 57(7): 2513-2528

63. Kyrou I, Schisler JC, Lang CH, et al. Chapter 7-fat hormones, adipokines, in endocrinology of the heart in health and disease. Academic Press 2017; p. 167-205.

64. Sagnelli D, Chessa S, Mandalari G, et al. Low glycaemic index foods from wild barley and amylose-only barley lines. J Funct Foods 2018; 40: 408-416.

65. Zhang X, Shen D, Fang Z, et al. Human gut microbiota changes reveal the progression of glucose intolerance. PLoS One 2013; 8(8): p. 71108.

66. Komaroff AL. The microbiome and risk for obesity and diabetes. JAMA 2017; 317(4): 355-356.

67. Perry RJ, Peng L, Barry NA, et al. Acetate mediates a microbiome-brain$\beta$-cell axis to promote metabolic syndrome. Nature 2016; 534(7606) 213-217.

68. Devaraj S, Hemarajata P and Versalovic J. The human gut microbiome and body metabolism: implications for obesity and diabetes. Clin Chem 2013; 59(4): 617-628.

69. Leite AZ, Rodrigues NDC, Gonzaga MI, et al. Detection of increased plasma interleukin-6 levels and prevalence of Prevotella Copri and Bacteroides Vulgatus in the feces of type 2 diabetes patients. Front Immunol 2017; 8: 1107-1107.

70. Ahmad A, Yang W, Chen G, et al. Analysis of gut microbiota of obese individuals with type 2 diabetes and healthy individuals. PloS one 2019; 4(12): 0226372-0226372.

71. Li Q, Chang Y, Zhang K, et al. Implication of the gut microbiome composition of type 2 diabetic patients from Northern China. Sci Rep $2020 ; 10(1):$ p. 5450

72. Han YW. Fusobacterium nucleatum: a commensal-turned pathogen. Curr Opin Microbiol 2015; 23:141-147.

73. Kaska L, Sledzinski T, Chomiczewska A, et al. Improved glucose metabolism following bariatric surgery is associated with increased circulating bile acid concentrations and remodeling of the gut microbiome. World J Gastroenterol 2016; 22(39): 8698-8719.

74. Ridlon JM, Kang DJ and Hyelemon PB, et al. Bile acids and the gut microbiome. Curr Opin Gastroenterol 2014; 30(3): 332-338.

75. Begley M, Gahan CGM, Hill C. The interaction between bacteria and bile. FEMS Microbiol Rev 2005; 29(4): 625-651.

76. Dawson PA and Karpen SJ. Intestinal transport and metabolism of bile acids. J Lipid Res 2015; 56(6): 1085-1099. 